\title{
Outdoor Sonar Sensing
}

\author{
Fernando J. Álvarez Franco ${ }^{1}$ and Jesús Ureña Ureña ${ }^{2}$ \\ ${ }^{1}$ University of Extremadura \\ ${ }^{2}$ University of Alcalá \\ Spain
}

\section{Introduction}

High-precision applications of airborne ultrasonic sensory systems have traditionally been restricted to indoor environments. The number of studies that have proposed the use of ultrasonic sensors outdoors is very limited and most times in a similar way that it has been made indoors. In applications like the guidance of vehicles and mobile robots, ultrasonic sensors are usually integrated in a more complex multi-sensory system, where they have been assigned low-precision tasks. Among these tasks are, for example, the detection of very close obstacles with which there exists an imminent risk of collision, or the coarse ranging of large navigation landmarks (Langer \& Thorpe, 1992), (Maeyama et al., 1994) (Guo et al., 2002).

Sonar's reputation as an unreliable sensing technology for outdoor applications is mainly due to the large influence that meteorological parameters have on the propagation of ultrasonic signals, which is a direct consequence of the mechanical nature of these waves. Changes in temperature and humidity, the presence of fog or rain in the atmosphere, and wind-induced refraction can cause strong variations in the attenuation of acoustic waves. As a result, a classical sonar system based on threshold detection of the signal envelope can provide very different results depending on the operating conditions. Furthermore, acoustic noise sources are more likely to be found outdoors. Aircrafts, pneumatic drills, bridge vibrations or even corona effects in high voltage cables are examples of ultrasonic sources which could render sonar in systems completely useless in certain environments. To overcome these problems, the research with ultrasonic sensors outdoors, has evolved to the use of special signal processing techniques such as the continuous transmission frequency modulated (CTFM) ultrasonic sensor used for path edge detection (Ratner \& McKerrow, 2003), the use of crosscorrelation with transmitted patterns for an outdoor sonar (Tanzawa et al., 1995) or the wind compensation method based on differential emitters for a positioning system (Jiménez \& Seco, 2005).

The first part of this chapter describes in detail the phenomenology associated with the propagation of acoustic waves in the atmosphere, placing an emphasis on the effects of the different mechanisms over the entire range of ultrasonic frequencies used in air. In the second part of the chapter, an outdoor sonar prototype is presented as an example of how the most recent signal coding and pulse compression techniques can be used to improve the reliability of sonar systems when operating under varying meteorological conditions. It will be seen that the transmission of ultrasonic encoded signals in the atmosphere entails a new 
challenge because of the effects of turbulence on the amplitude and phase of these signals. This problem is examined and results worthy of future exploration are pointed out in this new, fascinating, and open field of research.

\section{Propagation of ultrasonic waves in the atmosphere}

Sound propagation in the atmosphere has been the subject of intensive research since the second half of the twentieth century. This research has been mainly motivated by the increasing necessity to control noise generated by an ever more industrialized society.

There are several phenomena which have a significant effect on the propagation of acoustic waves through the atmosphere and these phenomena can be divided into three groups:

- Attenuation mechanisms

- Mechanisms affecting propagation speed

- Turbulence

Regarding the first group, useful conclusions for ultrasonic signals can be drawn by merely extrapolating the results already well established in the case of audio frequencies. A more detailed analysis is necessary when dealing with the other two categories.

\subsection{Attenuation mechanisms}

Geometrical spreading, atmospheric absorption and the attenuation caused by the presence of fog or rain are included in this group of phenomena.

Geometrical spreading is defined as the amplitude decay of an elastic wave caused by the expansion of its wave-front away from the source. Therefore, it does not depend on the propagation medium but on the features of the transducer employed. The acoustic field generated by a transducer with a certain level of symmetry in a point of spherical coordinates $(r, \phi, \theta)$ can be written as

$$
P(r, \varphi, \theta)=P_{a x}(r) \cdot D(\varphi, \theta)
$$

where $P_{a x}(r)$ is the acoustic pressure over the transducer $\left(P_{a x}(r)=P_{0} / r\right)$ and $D(\varphi, \theta)$ is the directional factor whose value depends on the symmetry that characterizes the transducer. For a typical circular piston-shaped transducer, the directional factor is given by the first order Bessel function of the first kind $J_{1}(\cdot)$ as

$$
D(\varphi, \theta)=D(\theta)=\frac{2 \cdot J_{1}(k \cdot a \sin \theta)}{k \cdot a \sin \theta}
$$

where $a$ is the piston radius and $k$ is the wave number $(k=2 \pi / \lambda)$. In the region close to the transducer axis, $D(\theta) \approx 1$ and the attenuation is that of spherical waves $P=P_{0} / r(6 \mathrm{~dB}$ loss when distance to the source is doubled).

In addition to the loss associated with geometrical spreading, when an acoustic wave propagates through the atmosphere part of its energy is dissipated into thermal energy causing the exponential decay of pressure with travelled distance. Thus, the acoustic field can be written as

$$
P(r, \varphi, \theta)=P_{a x}(r) \cdot D(\varphi, \theta) \cdot e^{-\alpha_{a b s} \cdot r}
$$


where $\alpha_{a b s}$ is the absorption coefficient, whose value depends on the mechanisms involved in this phenomenon. Air is one of the polyatomic gases which has been the subject of more in-depth study, and currently it is known that there are basically two different mechanisms that cause absorption of acoustic waves: the viscothermal losses (or classical absorption) and the oxygen and nitrogen molecular relaxation processes. The theoretical analysis of both processes led to a set of equations that have been later experimentally adjusted to increase agreement with real data. Today, these equations are grouped into the ISO-9613 standard (ISO, 1993). This rule establishes that the absorption coefficient can be calculated as

$$
\begin{aligned}
& \alpha_{a b s}(\mathrm{~Np} / \mathrm{m})=f^{2}\left\{18.4 \cdot 10^{-12}\left(\frac{P}{P_{r}}\right)^{-1}\left(\frac{T}{T_{20}}\right)^{\frac{1}{2}}+\right. \\
&\left.+\left(\frac{T}{T_{20}}\right)^{\frac{-5}{2}}\left[0.01275 \frac{e^{-2239.1 / T}}{f_{r o}+f^{2} / f_{r o}}+0.1068 \frac{e^{3352 / T}}{f_{r n}+f^{2} / f_{r n}}\right]\right\}
\end{aligned}
$$

where $f$ is the sound frequency in $\mathrm{Hz}, P$ is the atmospheric pressure in $\mathrm{Pa}\left(P_{\text {ref }}=101325 \mathrm{~Pa}\right)$, $T$ is the absolute temperature in $\mathrm{K}\left(T_{\text {ref }}=293.15 \mathrm{~K}\right)$, and $f_{r \mathrm{O}}, f_{r N}$ represent the relaxation frequencies of oxygen and nitrogen, respectively. As can be seen from Eq. (4), atmospheric absorption of acoustic waves depends on four parameters: wave frequency, temperature, humidity and pressure, although the dependence with the latter parameter is negligible if one takes into account the range of variation of this magnitude in practice. Fig. 1(a) shows the dependence of $\alpha$ with frequency for $T=20^{\circ} \mathrm{C}, H=70 \%$ and $P=1 \mathrm{~atm}$, together with the individual contribution of the different phenomena involved in this process. As can be seen, atmospheric absorption increases rapidly with frequency, being the vibrational relaxation of oxygen the dominant mechanism between $2 \mathrm{kHz}$ and $100 \mathrm{kHz}$. Fig. 1(b) shows the dependence of $\alpha$ with temperature and humidity for a fixed frequency of $50 \mathrm{kHz}$ and a pressure of $1 \mathrm{~atm}$. The values shown in this figure range between a minimum of $0.37 \mathrm{~dB} / \mathrm{m}$ for $T=-20^{\circ} \mathrm{C}$ and $H=0 \%$, and a maximum of $2.55 \mathrm{~dB} / \mathrm{m}$ for $T=50^{\circ} \mathrm{C}$ and $H=13 \%$. In the design of an ultrasonic sensory system intended for outdoor operation, one must take into account that the signal absorption in a warm summer afternoon can be more than six times greater than that of a cold winter morning.

Geometrical spreading and atmospheric absorption are always present when an acoustic signal is transmitted through the air. However, when this transmission is performed outdoors, there are other phenomena such as the presence of rain, fog or turbulence that can cause an additional attenuation of these signals. The effect of turbulence deserves special attention, and will be analysed later in this section. As for the other phenomena, a theoretical expression that can be used to estimate the attenuation caused by fog is that obtained by Cole and Dobbins (Cole and Dobbins, 1970):

$$
\alpha_{f o g}=k \cdot C_{m}\left\{\frac{\left(C_{4}-C_{8}\right)+\left(C_{1}-C_{6}\right) \frac{C_{5}}{C_{2}} \bar{\tau}_{t}^{2}}{C_{2} \bar{\tau}_{t}\left[1+\left(\frac{C_{5}}{C_{2}} \bar{\tau}_{t}\right)^{2}\right]}+\frac{\bar{\tau}_{d}}{1+\bar{\tau}_{t}^{2}}\right\} \quad(\mathrm{Np} / \mathrm{m})
$$


where coefficients $C_{i}$ are thermodynamic parameters depending on temperature, $k$ is the wave number, $C_{m}$ is the ratio between the drops mass and the mass of the gaseous phase per unit volume, and $\tau_{t}, \tau_{d}$ are adimensional relaxation times characterizing the processes of energy and momentum interchange responsible for the attenuation -see (Cole and Dobbins, 1970) for details-. These last three parameters depend on the droplet radius $R$ and on the number of droplets per unit volume $N$ :

$$
C_{m}=\frac{4 \pi R^{3} N \rho_{l}}{3 \rho_{m}} \quad \bar{\tau}_{t}=\frac{\omega R^{2} \rho_{l}}{3 \eta_{l} \rho_{m}} \quad \bar{\tau}_{d}=\frac{2 \omega R^{2} \rho_{l}}{9 v \rho_{m}}
$$

where $\rho_{l}$ is the droplets density, $\rho_{m}$ is the gaseous phase density, and $\eta_{l}$ and $v$ are the thermal diffusivity and cinematic viscosity coefficients of air respectively. From these expressions it can be concluded that, at a given temperature, the attenuation undergone by an acoustic wave propagating in a foggy atmosphere depends basically on three parameters: the droplets concentration, their radius and the wave frequency. In a given fog ( $R$ and $N$ constants), this attenuation increases with frequency until it reaches a saturation value that remains stable for higher frequencies. Since atmospheric absorption increases with the frequency to the power of two, a limit frequency exists above which atmospheric absorption is the dominant mechanism. Fig. 2 shows the attenuation values given by Eq. (5) in the range of frequencies $100 \mathrm{~Hz}-100 \mathrm{kHz}$ for $T=23^{\circ} \mathrm{C}$. Two types of fog are represented in this figure, a dense fog $\left(N=2000\right.$ drops $/ \mathrm{cm}^{3}$ with $\left.R=6 \mu \mathrm{m}\right)$ and a light fog $\left(N=400\right.$ drops $/ \mathrm{cm}^{3}$ with $R=10 \mu \mathrm{m}$ ). The attenuation associated with atmospheric absorption (Eq. (1)) has also been included in the same figure. Clearly, the dominant mechanism above $10 \mathrm{kHz}$ is atmospheric absorption, although a dense fog can cause an additional attenuation as large as $\approx 0.1 \mathrm{~dB} / \mathrm{m}$ in this range of frequencies.

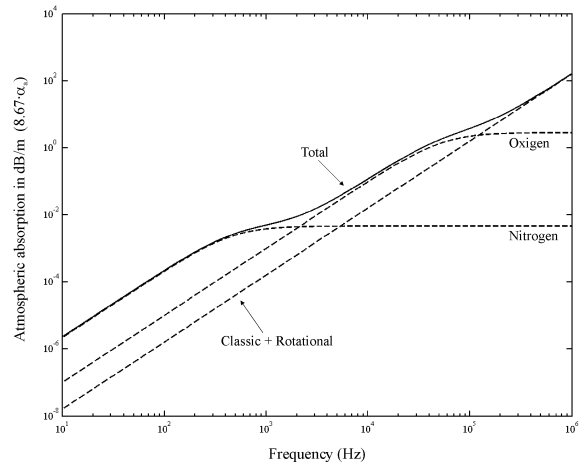

(a)

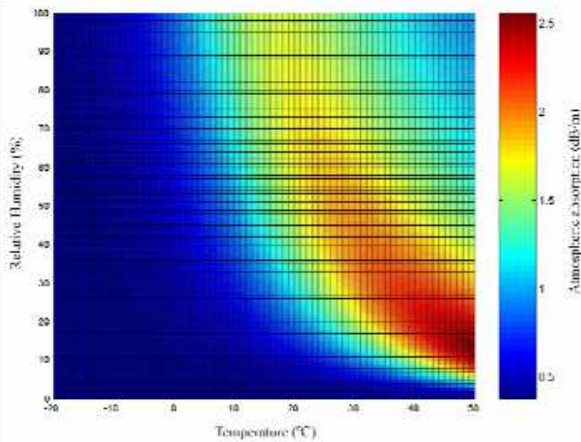

(b)

Fig. 1. Dependence of the atmospheric absorption coefficient $\alpha_{a b s}$ on different parameters. (a) Dependence on frequency for fixed temperature $\left(T=20^{\circ} \mathrm{C}\right)$, humidity $(H=70 \%)$ and pressure $(P=1 \mathrm{~atm})$. (b) Dependence on temperature and humidity for fixed frequency $(f=$ $50 \mathrm{kHz})$ and pressure and $(P=1 \mathrm{~atm})$.

With respect to rain, the drop size is so large in this case that the acoustic wave propagates through it without significant perturbation. Only a very intense rain can cause an appreciable attenuation. For frequencies greater than $20 \mathrm{kHz}$ Shamanaeva (Shamanaeva, 
1988) provides a very simple expression for the attenuation as a function of frequency and rain intensity $I(\mathrm{~mm} / \mathrm{h})$ :

$$
\alpha_{\text {rain }}=1.63 \cdot 10^{-28} f^{4.9} I^{1.5}(\mathrm{~Np} / \mathrm{m})
$$

Figure 2 also shows the results provided by Eq. (7) for an intense rain of $80 \mathrm{~mm} / \mathrm{h}$ and a light rain of $5 \mathrm{~mm} / \mathrm{h}$. As can be seen, an intense rain can cause in a $50 \mathrm{kHz}$ ultrasonic wave an attenuation similar to that caused by a dense fog $(\approx 0.1 \mathrm{~dB} / \mathrm{m})$, and this attenuation is even greater for higher frequencies, although it is always less than atmospheric absorption. For frequencies below $50 \mathrm{kHz}$ or in less intense rains this attenuation is negligible in practice.

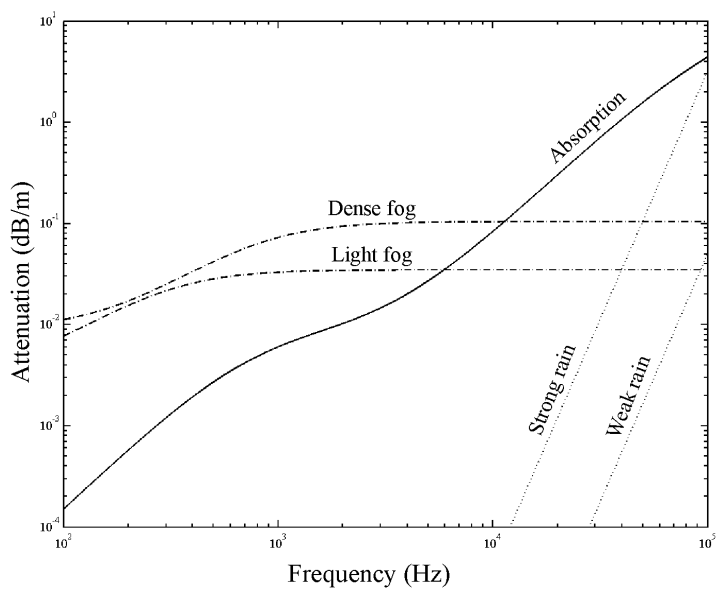

Fig. 2. Attenuation caused by the presence of fog and rain as a function of frequency.

\subsection{Mechanisms affecting the propagation speed}

Temperature and wind are the two meteorological parameters that have a stronger influence on sound speed propagation, whose apparent value $s$ can be given as:

$$
s=v_{p}+c(T) \cdot \sqrt{1-\left(\frac{v_{n}}{c(T)}\right)^{2}}
$$

where $c(T)$ is the propagation speed of the undulatory phenomenon (temperature dependent); and $v_{p}$ and $v_{n}$ are respectively, the parallel and normal to the direction of propagation components of wind which is responsible for convective transportation. Assuming that the normal component of the wind $v_{n}$ is small compared to the wave speed $c$, a reasonable approximation for the apparent speed is

$$
s \approx v_{p}+c(T)=v_{p}+331.6 \cdot \sqrt{1+\frac{T}{273.15}}
$$

where $T$ is the temperature in Celsius. In the atmosphere, both temperature and wind speed are functions that are strongly dependent on height, a dependency inherited by sound speed 
that causes the refraction of ultrasonic waves propagating outdoors. Figure 3 shows the effects of temperature-induced refraction. During the day the ground is heated by solar radiation, and this heat is transferred to the lower layers of air that progressively cool with increasing height $z$. This negative gradient of temperature causes the upward curvature of the acoustic rays, a situation depicted in Fig. 3a. The opposite situation occurs at night, when the ground rapidly cools by radiation and air temperatures are colder near the ground. In this case, the acoustic rays are bent downwards as shown in Fig. 3b.

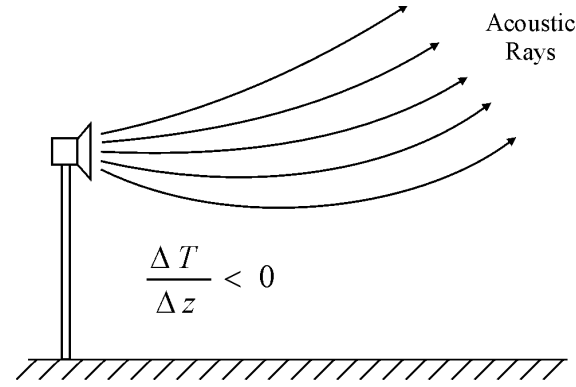

(a)

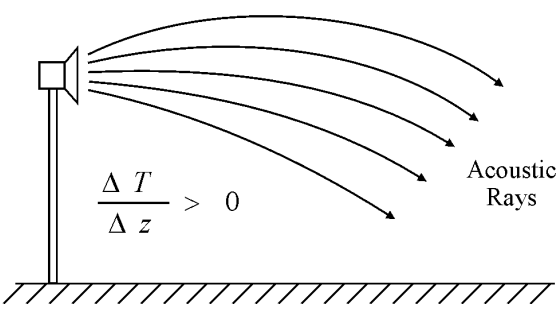

(b)

Fig. 3. Temperature-induced refraction.

A similar effect is caused by wind speed dependence on height. Due to the friction between the ground and the moving air, wind speed increases from zero at ground level to a practically constant value at an altitude of several hundreds of meters, a phenomenon known as wind shear. According to Eq. (9) an acoustic wave propagating downwind will be bent downwards, because the apparent sound speed is smaller, and the opposite effect will occur with a wave propagating upwind. Both situations are depicted in Fig. 4.

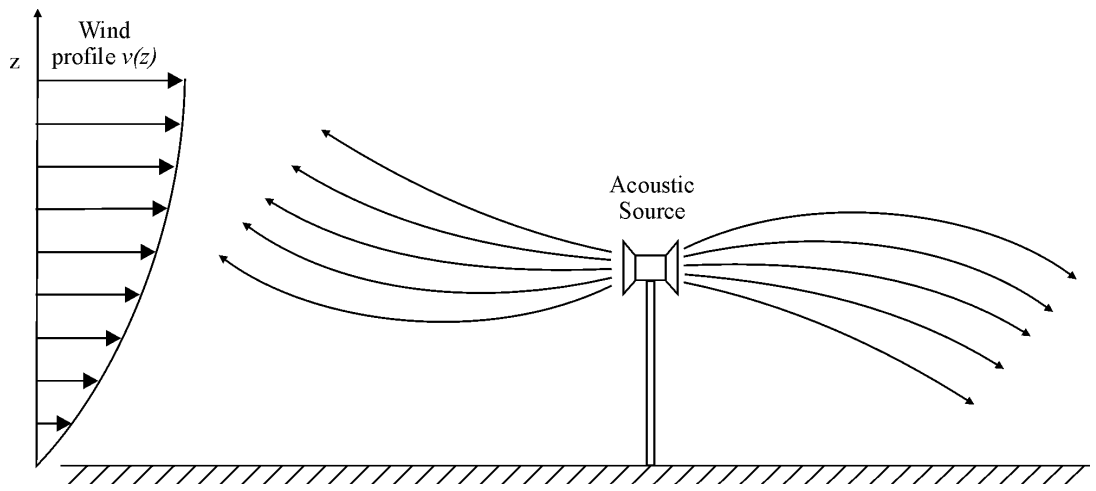

Fig. 4. Wind-induced refraction.

The quantitative analysis of this phenomenon requires an expression for the apparent sound speed as a function of height, $s=s(z)$. Then, it will be possible to calculate the trajectory followed by an acoustic ray using Snell's law

$$
\cos \theta=\frac{s(z)}{s_{0}}
$$


where $s_{0}$ represents the propagation speed at a reference height and $\theta$ is the angle between the acoustic ray and the horizontal plane. The expression for $s(z)$ can be obtained from Eq. (9) if the profiles for temperature $T(z)$ and wind velocity $v(z)$ are known. In the surface layer of the atmosphere, whose height may vary from $10 \mathrm{~m}$ on clear nights to $100 \mathrm{~m}$ on windy days, these profiles can be obtained from the Monin-Obukhov similarity theory (Monin \& Obukhov, 1954):

$$
\begin{gathered}
T(z)=T\left(z_{0}\right)+\frac{T^{*}}{K_{a}}\left[\ln \left(\frac{z}{z_{0}}\right)-\psi_{T}\left(\frac{z}{L_{m o}}\right)\right]-\frac{G_{0}}{c_{P}}\left(z-z_{0}\right) \\
v_{l}(z)=\frac{v^{*}}{K_{a}}\left[\ln \left(\frac{z}{z_{0}}\right)-\psi_{v}\left(\frac{z}{L_{m o}}\right)\right]
\end{gathered}
$$

In these expressions $K_{a}$ is Von Karman's constant, whose value is approximately $0.4 ; c_{p}$ is the air specific heat at constant pressure; and $G_{0}$ is the gravitational acceleration module. Constants $T^{*}$ and $v^{*}$ represent two scale values for temperature and wind speed respectively and their values are usually experimentally adjusted. Constant $z_{0}$ is the roughness length, and it is a measure of the minimum height below which the profiles above are not valid. Its value depends on the roughness characteristics of the terrain and can be obtained with reasonable accuracy from tables by simple inspection of the terrain - see for example (Panofsky \& Dutton, 1984) -

Functions $\psi_{T}$ and $\psi_{v}$ appearing in the equations above depend on the type of atmosphere. These functions have been empirically adjusted to obtain the following expressions:

$$
\begin{gathered}
\psi_{T}\left(\frac{z}{L_{m o}}\right)=\left\{\begin{array}{cc}
2 \ln \left[0.5 \cdot\left(1+x^{2}\right)\right] & \text { Unstable } \\
0 & \text { Neutral } \\
-5 \frac{z}{L_{m o}} & \text { Stable }
\end{array}\right. \\
\psi_{v}\left(\frac{z}{L_{m o}}\right)=\left\{\begin{array}{cc}
\ln \left[\left(\frac{1+x^{2}}{2}\right)\left(\frac{1+x}{2}\right)^{2}\right]-2 \arctan x-\frac{\pi}{2} & \text { Unstable } \\
0 & \text { Neutral } \\
-5 \frac{z}{L_{m o}} & \text { Stable }
\end{array}\right.
\end{gathered}
$$

being $x=\left(1-16 z / L_{m o}\right)^{1 / 4}$.

Finally, $L_{m o}$ is the Monin-Obukhov length that basically depends on the vertical flux of heat at the surface. Since this flux cannot be easily measured experimentally, $L_{m o}$ is not directly calculated in practice. Instead, its value is inferred from the relation of this parameter with the roughness length and Turner classes found by Golder (Golder, 1972). Turner classes constitute a classification method for different types of atmospheres, which is based on the relative importance that thermal convection and mechanical turbulence have on a particular atmosphere. Seven classes exist numbered from 1 to 7 . The first three classes correspond to 
unstable atmospheres, where thermal convection prevails over mechanical turbulence (sunny days with light winds), class 4 represents neutral atmospheres where thermal convection does not exist and only mechanical turbulence is present (days and nights with strong winds), and the last three classes correspond to stable atmospheres characterized by an inversion of the temperature gradient (clear nights with light winds). The class a certain atmosphere belongs to, may be directly calculated from the solar altitude (which determines the intensity of the radiation received), the cloud cover, and the wind speed at a reference height. The relation experimentally obtained by Golder between $z_{0}, L_{m o}$ and Turner classes is represented in Fig. 5

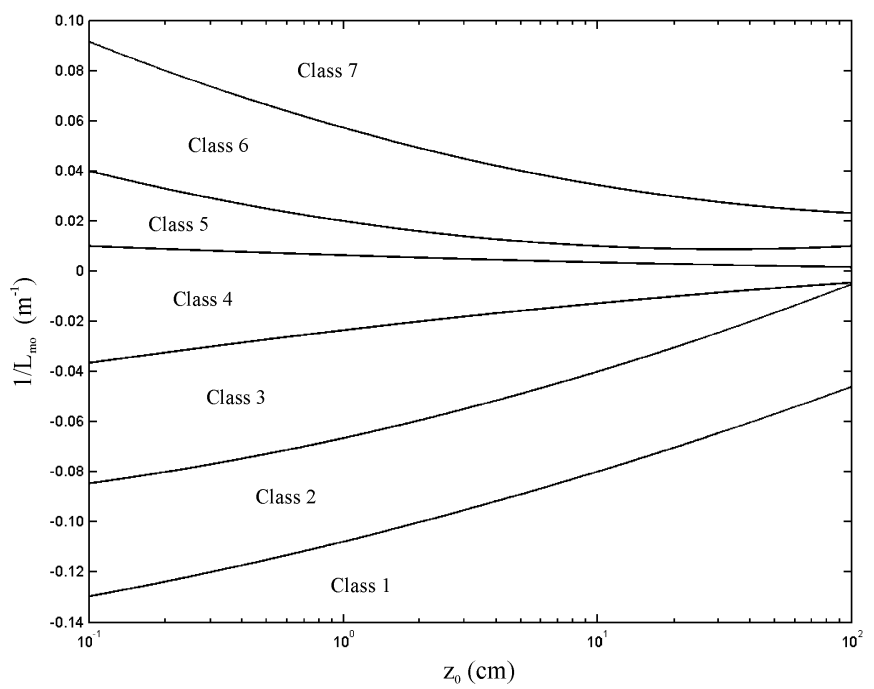

Fig. 5. Relation between $z_{0}, L_{m o}$ and Turner classes (Golder, 1972).

Let us now focus on the influence that this phenomenon has on the signal emitted by a sonar system. Due to atmospheric absorption, a signal horizontally emitted can cover a maximum distance of several tens of meters before reaching an object, and the maximum vertical deviation caused by refraction is not expected to exceed a few meters even in the most unfavorable conditions. In such a situation, it can be generally assumed that the apparent sound speed depends linearly on height, and then, the departure angle of a ray that is detected by a receiver placed at a distance $r$ from the emitter in the same horizontal plane is given by:

$$
\theta_{s}=\arctan \left(\frac{r \cdot g}{2 \cdot s_{0}}\right)
$$

where $g=d s / d z$ is the constant speed gradient and $s_{0}$ is the apparent sound speed at the height of the sensor. The constant gradient can be obtained as the average sound speed variation in the first Fresnel ellipsoid. This ellipsoid is represented in Fig. 6, and it is defined as the region of the space where the differences between the length of the direct path and the length of any diffracted path is less than a half wavelength: 


$$
r_{1}+r_{2}-r=\frac{\lambda}{2}
$$

From this figure, the constant gradient can be expressed as:

$$
g=\frac{s\left(h_{\max }\right)-s\left(h_{\min }\right)}{h_{F}}
$$

where $h_{F}$ represents the ellipsoid height, which depends both on the wavelength $\lambda$ and on the emitter-receiver distance $r$ as shown

$$
h_{F}=\sqrt{\frac{\lambda}{4} \cdot\left(r+\frac{\lambda}{4}\right)}
$$

The evaluation of Eq. (13) is a tedious task which involves many previous calculations that are necessary to obtain the constant speed gradient $g$. An algorithm performing all these calculations has been used in (Alvarez et al., 2008) to study a particular ultrasonic system installed at a height of $150 \mathrm{~cm}$ over a terrain a short grass where maximum wind speeds of $7.6 \mathrm{~m} / \mathrm{s}$ were registered during a period of 6 months. This algorithm predicted a maximum departure angle of $1.6^{\circ}$ under the worst conditions of wind, assuming a propagation distance of $14 \mathrm{~m}$. This angle causes a negligible additional attenuation of $0.2 \mathrm{~dB}$ in the signal received at the same height if a Polaroid series 600 transducer (Polaroid, 1999) is employed in the emission.

This conclusion should not be generalized, though. If transducers with narrower emission patterns are used, longer propagation distances are considered, or if the system is installed in locations where greater speed gradients can occur then the effect of refraction might not be insignificant.

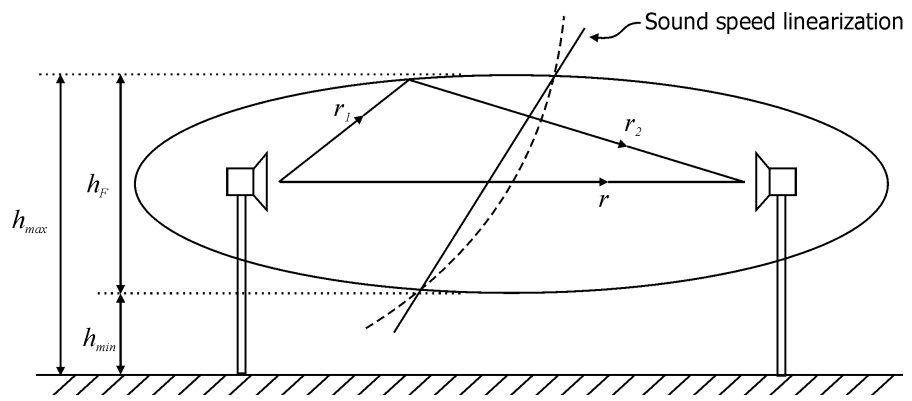

Fig. 6. Fresnel ellipsoid.

\subsection{Turbulence}

Wind is rarely stationary in the lower layers of the atmosphere and almost always random fluctuations of its behavior appear in the form of highly rotational fluxes. These turbulent eddies cause local random fluctuations in wind velocity and temperature, two parameters that have a strong influence on the propagation velocity of acoustic waves as was seen above. Each one of these eddies causes a sudden change on the refraction index and the subsequent scattering of part of the wave energy. An immediate consequence of this scattering is the additional attenuation undergone by a wave propagating through a 
turbulent medium. The average value of this attenuation was obtained by Brown and Clifford (Brown \& Clifford, 1976) as

$$
A_{\text {turb }}=10 \log \left(1+0.48 k^{12 / 5} d_{0}^{2} r^{6 / 5} C_{n}^{12 / 5}\right) \quad(\mathrm{dB})
$$

where $k$ is the wave number; $d_{0}$ is the diameter of the transducer; $r$ is the travelled distance and $C_{n}^{2}$ is the refractive index structure parameter which characterizes the turbulence strength. Equation (17) provides attenuation values far below those associated with atmospheric absorption in the range of frequencies $100 \mathrm{~Hz}-100 \mathrm{kHz}$, as shown in Fig. 7.

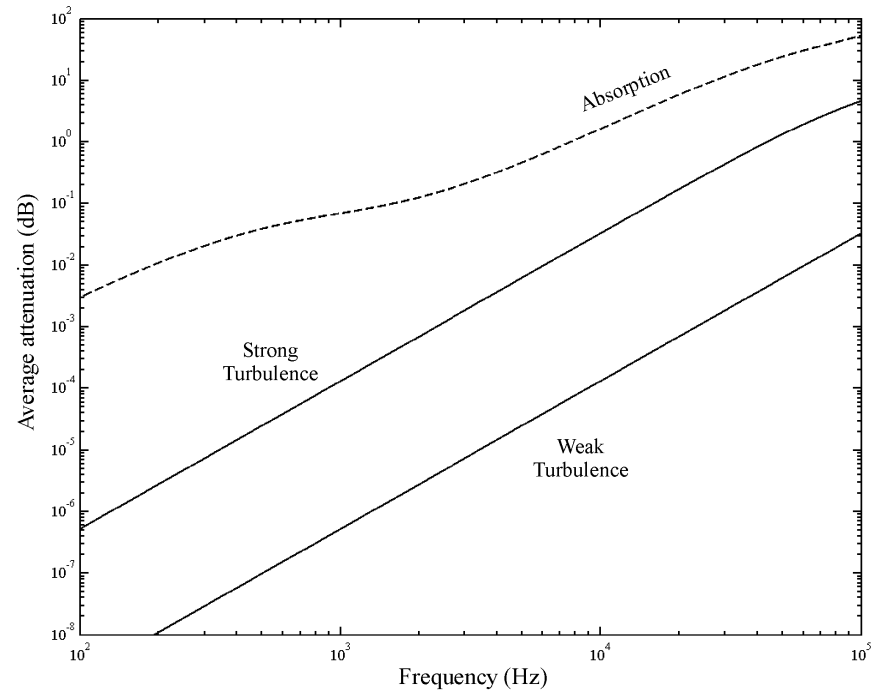

Fig. 7. Attenuation associated with the presence of turbulence as a function of frequency.

Nevertheless, it is important to note that this attenuation is an average value and that the instantaneous values can vary widely around it. When an acoustic wave propagates through a turbulent region, it encounters a variety of eddies with different sizes, velocities and temperatures. Their combined effect alters the initial coherence of the wavefronts, which will no longer be spherical nor have identical amplitude after crossing the turbulent region. This situation is depicted in Fig. 8. A receiver placed at a certain distance from the emitter will record random fluctuations in the amplitude and phase of the acquired signals. This effect can be characterized through a coherence time $t_{c}$, defined as the time during which the characteristics of an acoustic wave propagating through a turbulent region remain essentially invariant.

Turbulence theory provides a means to estimate the value of this time theoretically. Random fluctuations of wind velocity and temperature in a turbulent atmosphere cannot be described as stationary random fields through the spatial correlation function

$$
C_{u}\left(r_{1}, r_{2}\right)=\left\langle\left[u\left(r_{1}\right)-\left\langle u\left(r_{1}\right)\right\rangle\right] \cdot\left[u\left(r_{2}\right)-\left\langle u\left(r_{2}\right)\right\rangle\right]\right\rangle
$$

where $u$ represents a generic magnitude; $<\bullet>$ is the spatial average operator; and $r_{1}, r_{2}$ two arbitrary positions. These meteorological fields do not have constant mean and Eq. (18) is 
not invariant under translations. In this case, the three dimensional behavior is best described through the so-called structure functions, first introduced by Kolmogorov (Kolmogorov, 1941)

$$
D_{u}\left(r_{1}, r_{2}\right)=\left\langle\left\{\left[u\left(r_{1}\right)-\left\langle u\left(r_{1}\right)\right\rangle\right]-\left[u\left(r_{2}\right)-\left\langle u\left(r_{2}\right)\right\rangle\right]\right\}^{2}\right\rangle
$$
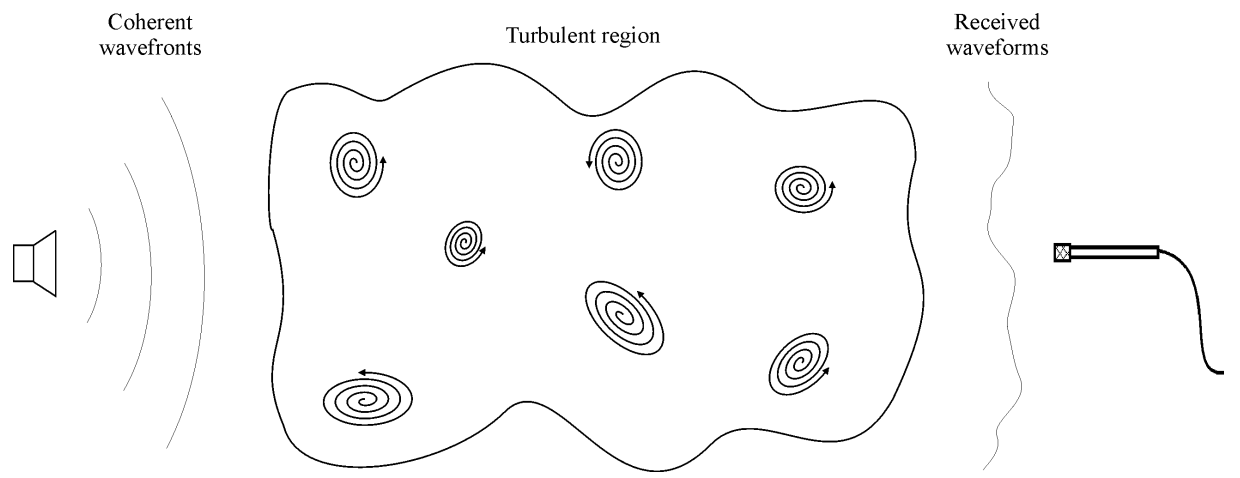

Fig. 8. Propagation of an acoustic wave through a turbulent medium.

Although the statistical properties of these random fields depend on location, it can be assumed that difference between values taken at any two points depends on the distance $\left|r_{1}-r_{2}\right|$ whenever this distance is not excessively large, a property known as local homogeneity. Then, if isotropy is also assumed Eq. (19) takes the simpler form:

$$
D_{u}(r)=\left\langle\left[u\left(r_{0}+r\right)-u\left(r_{0}\right)\right]^{2}\right\rangle
$$

This structure function is related to the three dimensional power spectrum $\Phi_{u}(k)$ (Fourier transform of the correlation function) according to the following expression

$$
\Phi_{u}(k)=\frac{1}{4 \pi^{2} k^{3}} \int_{0}^{\infty} \frac{\sin (k r)}{k r} \frac{d}{d r}\left[r^{2} \frac{d}{d r} D_{u}(r)\right] d r
$$

where $k=2 \pi / \lambda$ is the spatial wavenumber. The spectral analysis of random fields in a turbulent medium is very useful because such a regime can be characterized by several length scales. The longer scale is named outer scale or integral scale $L_{0}$ and represents the size of the larger eddies in the atmosphere. These eddies are very energetic and low dissipative, and their size is in the range of some tens of meters, although it may vary according to local conditions. The energy of these eddies is redistributed without loss to eddies of decreasing size until it is converted into heat by viscous dissipation (see Fig. 9). The size of the smaller eddies is named inner scale or microscale $l_{0}$ and it measures approximately a few milimeters. Taking into account that the amount of kinetic energy per unit mass is proportional to $v^{2}$, and assuming that the rate of transfer of energy from the largest eddies of size $L$ is proportional to $v / L$, then the rate of energy supply to the small-scale eddies is in the order of $v^{3} / L$. The range of distances between the outer scale and the inner scale is named the inertial subrange and, as the energy is transported from large eddies to small eddies in this range 
without piling up at any scale, the rate of energy supply must be equal to the dissipation rate $\varepsilon$, and then $v^{2} \alpha L^{2 / 3}$. This dimensional analysis was carried out by Kolmogorov who concluded that in the inertial subrange the structure functions of some meteorological magnitudes such as wind velocity, temperature and refraction index must exhibit the same dependence on distance, i.e

$$
D_{u}(r)=C_{u}^{2} \cdot r^{2 / 3}
$$

$\mathrm{C}_{u}{ }^{2}$ is a constant called structure parameter of magnitude $u$ fluctuations, and it depends on the turbulence strength. Combining Eq. (22) and Eq. (21) the Kolmogorov power spectrum is obtained

$$
\Phi_{u, K}(k)=0.033 \cdot C_{u}^{2} \cdot k^{-11 / 3}
$$

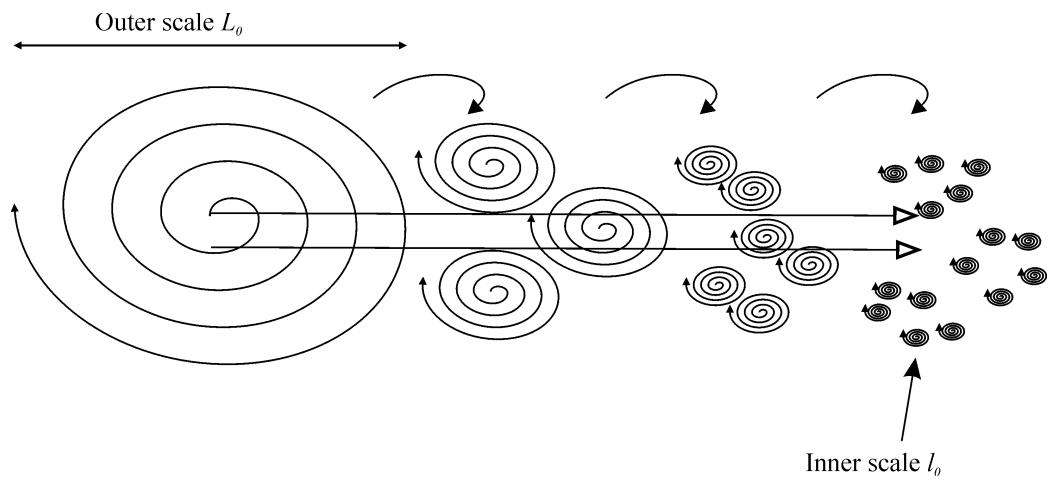

Fig. 9. Kolmogorov model of Turbulence.

The spatial coherence of an acoustic wave is usually described by means of the mutual coherence function $(M C F)$, defined as the cross-correlation function of the complex pressure field in a plane perpendicular to the direction of propagation

$$
\operatorname{MCF}(\boldsymbol{\rho}, r)=\left\langle P(\mathbf{r}+\boldsymbol{\rho}, r) \cdot P^{*}(\mathbf{r}, r)\right\rangle
$$

where $\rho$ is the separation along the plane that is perpendicular to the direction of propagation and located at a distance $r$ from the source. Tatarskii (Tatarskii, 1971) showed that a simple relation exists between the MCF and the phase and log-amplitude structure functions given by $D_{\Phi}$ and $D_{\chi}$ respectively

$$
\operatorname{MCF}(\boldsymbol{\rho}, r)=\exp \left\{-\frac{1}{2}\left[D_{\chi}(\boldsymbol{\rho}, r)+D_{\phi}(\boldsymbol{\rho}, r)\right]\right\}
$$

From this expression, and assuming that scattering angles are small, Tatarskii obtained the following expression valid for spherical waves propagating in an isotropic medium

$$
M C F(\boldsymbol{\rho}, r)=\exp \left\{-4 \pi^{2} k^{2} r \times \int_{0}^{\infty}\left[1-\int_{0}^{1} J_{0}(K \rho u) d u\right] \phi_{n}(K) K d K\right\}
$$


where $J_{0}$ is the zero order Bessel function of the first kind. A further simplification of Eq. (26) requires selecting a model for atmospheric turbulence through the power spectrum of the refraction index $\Phi_{n}(k)$. The model to be selected is determined by the size of the eddies that have a larger scattering effect, which are the ones whose size is about one Fresnel Zone $(\lambda \cdot r)^{1 / 2}$. For an ultrasonic wave of about $50 \mathrm{kHz}$ traveling over some tens of meters, the Fresnel Zone is clearly within the inertial region and $\Phi_{n}(k)$ must be selected as a Kolmogorov spectrum (Eq. (23)). Introducing Eq. (23) into Eq. (26) provides a method to measure the size of the coherent wavefront as the lateral separation value $\rho_{0}$ where the MCF is $1 / e$ times lower than its on-axis value, i.e. $\operatorname{MCF}\left(\rho_{0,}, r\right)=1 / e$. This length is called lateral coherence length and can be calculated as follows

$$
\rho_{0}=\left(0.545 \cdot k^{2} \cdot C_{n}^{2} \cdot r\right)^{-3 / 5} \quad r_{i}<r<r_{c}
$$

where the lengths $r_{c}$ and $r_{i}$ defining the range of validity of the above expression are given by

$$
\begin{gathered}
r_{c}=\left[0.4 k^{2} C_{n}^{2}\left(L_{0} / 2 \pi\right)^{5 / 3}\right]^{-1} \\
r_{i}=\left[0.4 k^{2} C_{n} l_{0}^{5 / 3}\right]^{-1}
\end{gathered}
$$

These expressions were first obtained by Yura (Yura, 1971) for optical waves, but they are still valid for acoustic if the structure parameter of the refraction index is replaced with a new efficient structure parameter defined as (Ostashev, 1997)

$$
C_{n}^{2}=\frac{C_{T}^{2}}{T^{2}}+\frac{22}{3} \frac{C_{v}^{2}}{c^{2}}
$$

where $T$ is the temperature; $c$ is the sound speed; and $C_{T^{2}}, C_{v}{ }^{2}$ represent the structure parameters of temperature and wind velocity respectively.

From the lateral coherence length, it is possible to estimate the temporal coherence of a received wave if a "Frozen Model" for the turbulent atmosphere is assumed. This model establishes that in a turbulent atmosphere the collection of eddies remains frozen in relation to one another while the entire collection moves with mean wind velocity $v$. In this case a direct relation exists between the spatial and temporal behaviors of the statistical fields:

$$
u\left(\mathbf{r}, t+t^{\prime}\right)=u\left(\mathbf{r}-\mathbf{v} \cdot t^{\prime}, t\right)
$$

In a first approximation, the effects derived from the longitudinal displacement of the pattern are negligible when compared to those derived from the transversal one. Then, the time for which a signal received at a certain distance remains coherent is equal to the time that the pattern of the eddies takes to travel over the lateral coherence length. This coherence time is given by (Álvarez et al., 2006)

$$
t_{c}=\frac{1}{v_{n} \cdot\left(0.545 k^{2} C_{n}^{2} r\right)^{3 / 5}}
$$

where $v_{n}$ is the transversal component of the wind. For an ultrasonic signal of $50 \mathrm{kHz}$ and a reference propagation distance of $r=14 \mathrm{~m}$, this expression yields a minimum value of 8.2 
$\mathrm{ms}$ for the coherence time when strong turbulence conditions $\left(\mathrm{C}_{\mathrm{n}^{2}}=10^{-5} \mathrm{~m}^{-2 / 3}\right)$ and $v_{n}=10$ $\mathrm{m} / \mathrm{s}$ are assumed. For greater values of wind velocity small scattering angles cannot be assumed and Eq. (31) is no longer valid.

\section{Reliable outdoor operation: signal coding and pulse compression}

It is clear from the results obtained in the previous section that a classical sonar system whose echoes are detected when they or their envelopes first exceed a certain threshold, cannot reliably operate outdoors. The amplitude of the echo generated by the same object at the same distance can vary largely depending on meteorological conditions of temperature, humidity, rain, fog and wind, giving rise to very different measurements of time-of-flight (TOF). This problem could be partially overcome by adapting the detection threshold to the average attenuation associated to current values of these conditions. However, the random and wide variations of amplitude and phase induced by turbulence are very difficult to deal with.

Classical systems are very sensitive to ultrasonic noise too, since such noise is added to the received echo modifying the instant in which it or its envelope exceeds the threshold. High intensity noise could even be confused with real echoes giving rise to phantom reflectors (artifacts). Obviously, robustness to noise can be improved by increasing the energy of the emissions, but there is always a physical limit for the maximum amplitude than can be transmitted with a given transducer. If envelope detection is used, another alternative would be to increase the duration of the emissions, but at the expense of degrading the system precision (two overlapped echoes cannot be distinguished by these systems).

Signal coding and pulse compression techniques emerge as an attractive alternative in the development of reliable outdoor sonars. These techniques have been already used in the design of high performance indoor sonars that are capable of simultaneously measuring the TOF of echoes coming from different emissions with a precision of microseconds. Instead of ultrasonic pulses, these systems emit modulated binary codes with good correlation properties that are detected through matched filtering. Thus, a correlation peak is obtained only when the code matched to the corresponding filter is received, the relative height of this peak being proportional to the length (and not the amplitude) of the code. With these techniques the system has a resolution similar to that obtained with the emission of short pulses, still maintaining the high robustness to noise achieved with the emission of long pulses (hence the name pulse compression). Strong variations in the amplitude of the received echoes modify the height, but not the position, of the correlation peak and the results provided by the system would be the same regardless of the attenuation of the signal if the detection threshold for this peak is sensitive enough. Moreover, an adequate selection of codes with low values of cross-correlation allows a system composed of several transducers to perform simultaneous measurements under exactly the same operating conditions.

However, random fluctuations of amplitude and phase induced by turbulence are still a problem. If the coherence time that characterizes this phenomenon (Eq. (31)) is much shorter than the length of the emitted code, this code could be completely distorted before arriving at the receiver and it could not be properly detected by matched filtering. The following sections deal with the analysis of this phenomenon. 


\subsection{Outdoor prototype}

The outdoor propagation of encoded signals has been studied with the help of the prototype shown in Fig. (10). In this system, a Polaroid series 600 electrostatic transducer (Polaroid, 1999) placed 1.5 meters over the ground is used as the emitter. A high-frequency microphone placed at 14 meters from the emitter in the same horizontal plane has been used as the receiver. In order to minimize the filtering effect associated with the acoustic pattern peculiarities of both the emitter and the receiver, a laser pointer was used to align their axes. All the process has been controlled by a PC equipped with an acquisition board that simultaneously sends the emission pattern to the amplifier driving the transducer and acquires the signal coming from the microphone. Figure 10a shows a picture of the experimental site with the emitter in the background. This emitter, together with the anemometer, can be seen in more detail in Fig. 10b.

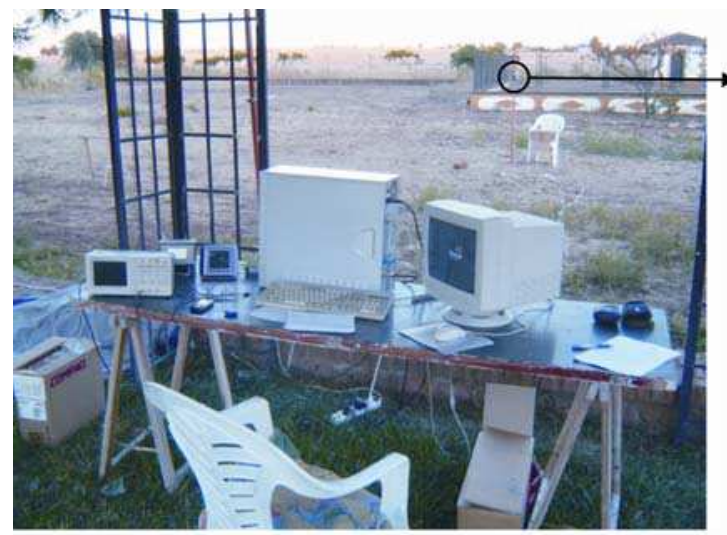

(a)

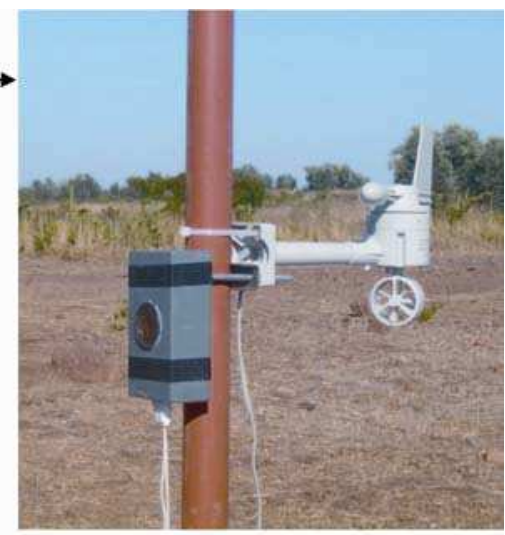

(b)

Fig. 10. Experimental site (a) and detailed view of the transducer with the anemometer (b).

This system has been used to conduct the emission of complementary sets of four sequences with different lengths. A set of 4 binary sequences $\left\{x_{i}[n], 1 \leq i \leq 4\right\}$, whose elements are either +1 or -1 , is a complementary set if the sum of their aperiodic autocorrelation functions $\phi_{x i x i}$ equals zero for all non-zero time shifts

$$
\phi_{x 1 x 1}[n]+\phi_{x 2 \times 2}[n]+\phi_{x 3 \times 3}[n]+\phi_{x 4 x 4}[n]=\left\{\begin{array}{cc}
4 \cdot L & n=0 \\
0 & n \neq 0
\end{array}\right.
$$

where $L$ is the length of the sequences. One main advantage that complementary sequences have against other codes used for pulse compression, such as Barker codes or m-sequences, is the existence of orthogonal families. Two sets with the same number of sequences $\left\{x_{i}[n]\right.$, $\left.y_{i}[n] ; 1 \leq i \leq 4\right\}$ are said to be orthogonal when the sum of the corresponding cross-correlation functions equals zero

$$
\phi_{x 1 y 1}[n]+\phi_{x 2 y 2}[n]+\phi_{x 3 y 3}[n]+\phi_{x 4 y 4}[n]=0 \quad \forall n
$$

This property allows the simultaneous emission of different signals with ideal null interference. The other main advantage of using these sequences is the existence of an 
efficient correlation system called Efficient Sets of Sequences Correlator (ESSC), that notably reduces the total number of operations carried out from $4 \times 2 \times(L-1)$ to $4 \times \log _{2} L$ in order to perform the correlations with the four sequences in a set (Álvarez et al., 2004). This digital filter, shown in Fig. 11, is formed by $\log _{2} L$ similar stages, each one with 3 delay elements and 8 adders/substracters. Coefficients $W_{i, j}$ appearing in this figure take values +1 or -1 and are not implemented as amplifiers in practice.

In our prototype, the four sequences composing the set have been simultaneously transmitted through the Polaroid transducer first by interleaving these sequences to generate a new $4 L$-bit sequence defined as:

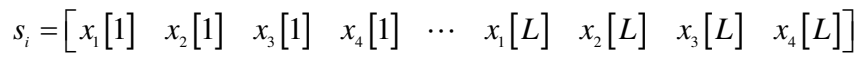

and then by implementing the BPSK modulation of the new sequence with a symbol formed of two cycles of a $50 \mathrm{kHz}$ carrier. The modulated signal has a centralized bandwidth of about $12.4 \mathrm{kHz}$ that allows its efficient transmission through the $\approx 20 \mathrm{kHz}$ bandwidth of this transducer. The total duration of the emission is proportional to the length of the sequences and is given by:

$$
t_{e}=4 L \text { (bits) } \times 2 \text { (cycles) } \times 20 \mu s=160 L \mu s
$$

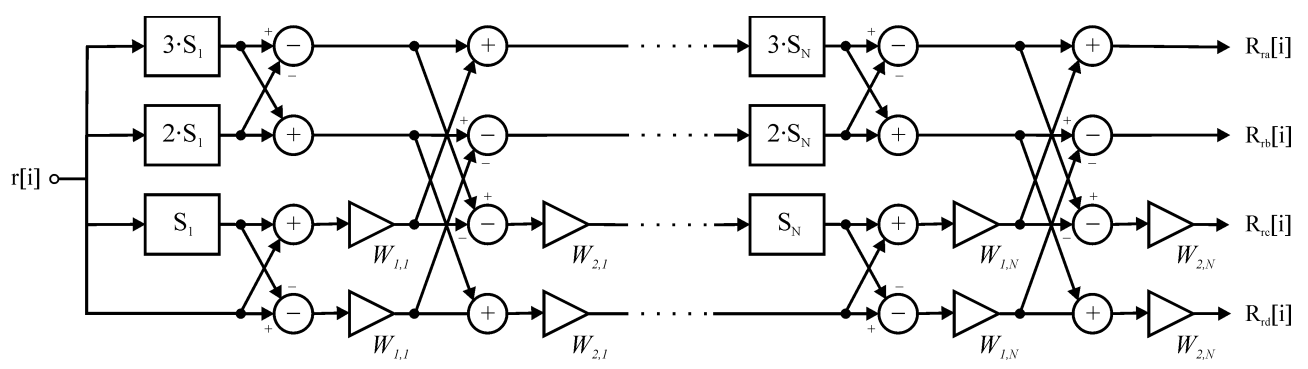

Fig. 11. Efficient Sets of Sequences Correlator (ESSC).

The signal received by the microphone placed at $14 \mathrm{~m}$ from the transducer is first sampled at a rate of $800 \mathrm{ksps}$, and then demodulated with a digital correlator matched to the modulation symbol (32 samples). Actually, this filter correlates the acquired signal with a binarized version of the symbol, simplifying the operations at the expense of a nearly negligible decrease in the output $S N R$. The signal from the demodulator is an interpolated version of the sequence obtained by interleaving the complementary sequences (Eq. (34)), with an interpolation factor of 32. Thus, in this signal, two samples belonging to the same sequence are $4 \times 32=128$ samples apart, and it is necessary to decimate the signal by the same factor prior to carrying out the correlations. This decimation can be easily achieved just by multiplying the values of all the delay stages in the ESSC by 128 . Finally, taking into account that the bits of the interleaved sequences are delayed 32 samples, it is necessary to add three additional delay stages at the outputs of the ESSC in order to perform the inphase sum of the autocorrelation functions. 
In the precise moment in which the last sample of the set matched to the correlator is acquired, a maximum value, ideally proportional to $\phi_{x 1 \times 1}[0]+\phi_{x 2 \times 2}[0]+\phi_{x 3 \times 3}[0]+\phi_{x 4 x 4}[0]$, is obtained at the input of the peak detector. However, as a consequence of the asynchronism characterizing the detection process, this maximum value always appears with self-induced noise depending on the shape of the modulation symbol. A parameter commonly used to measure the quality of this signal is the Sidelobe-to-Mainlobe Ratio (SMR), defined as the ratio between the higher value obtained outside the vecinity of the main peak and the value of this peak.

\subsection{Experimental results}

In order to investigate the effect of turbulence on the performance of the prototype described in the previous section, a continuous emission of the codes has been conducted. Fig. 12 shows the received and processed signals when complementary sequences of 64 bits $\left(t_{e}=10.24 \mathrm{~ms}\right)$ are emitted under very weak and very strong turbulent conditions. As can be seen in this figure, in both cases all the sets are properly detected, although the scattering of energy caused by turbulence is evident in Fig 12b, with the consequent deterioration of the $S M R$. Figure 13 shows the same signals when sequences of 256 bits ( $\left.t_{e}=40.96 \mathrm{~ms}\right)$ are emitted. As can be seen in Fig. 13a, under weak turbulent conditions the sets are still property detected, but when the coherence time is clearly shorter than the emission time spurious peaks appear that may confuse the system. In this case, when the received signal is compared to the emission pattern, slight compressions and expansions can be clearly visualized which are deteriorating the phase coherence required by the correlation process. This comparison is represented in Figs. 14 and 15 for very weak and very strong turbulence respectively.

The observed increase in $S M R$ has been experimentally studied under different conditions of turbulence, and the results are presented in Table 1. The SMR remains below 0.3 even under very strong turbulence when 64-bit sequences are transmitted, showing the good performance of the system in all cases. The same result is true with 256-bit sequences except
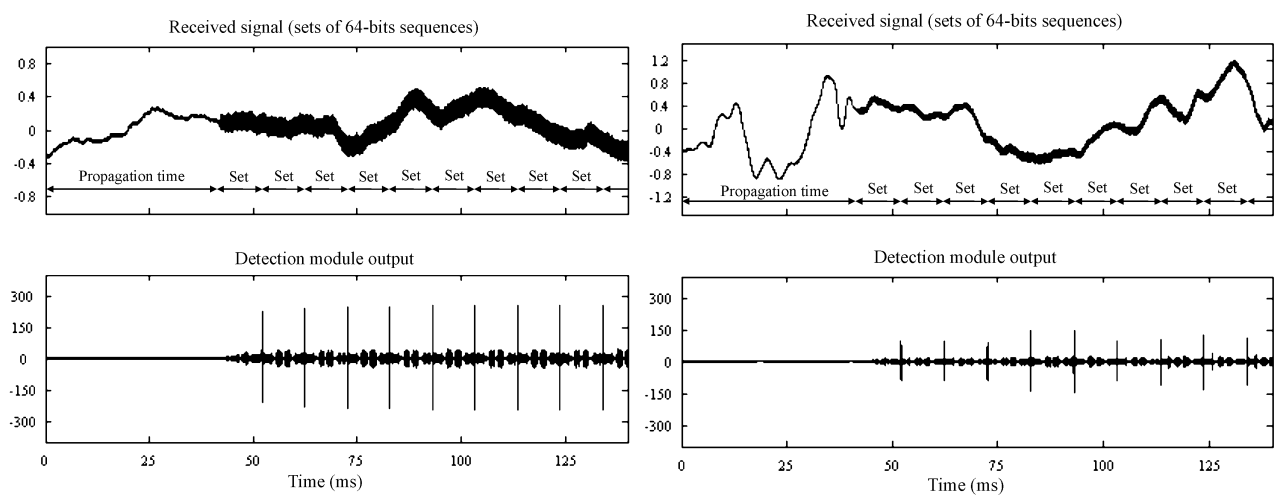

(a)

(b)

Fig. 12. Detection process of 64 -bit sequences $\left(t_{e}=10.24 \mathrm{~ms}\right)$ under very weak (a) and very strong (b) turbulence conditions. 

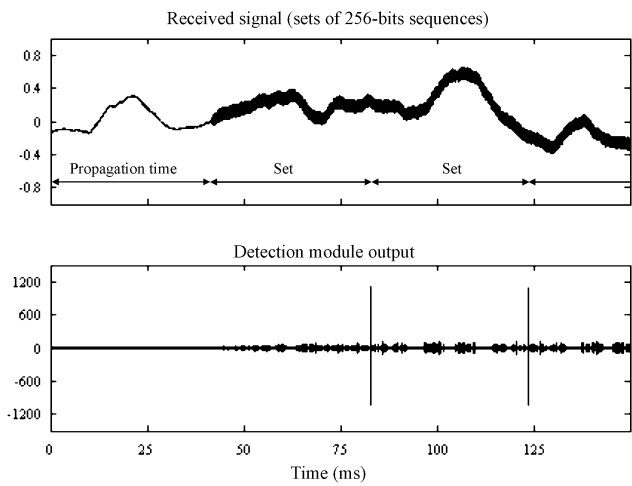

(a)
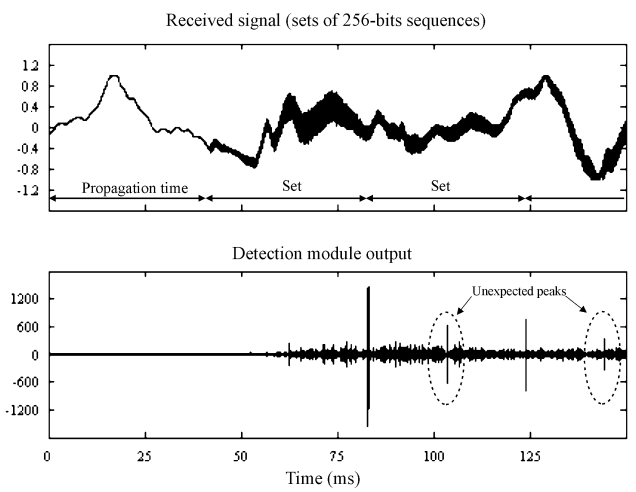

(b)

Fig. 13. Detection process of 256-bit sequences $\left(t_{e}=40.96 \mathrm{~ms}\right)$ under very weak (a) and very strong (b) turbulence conditions.

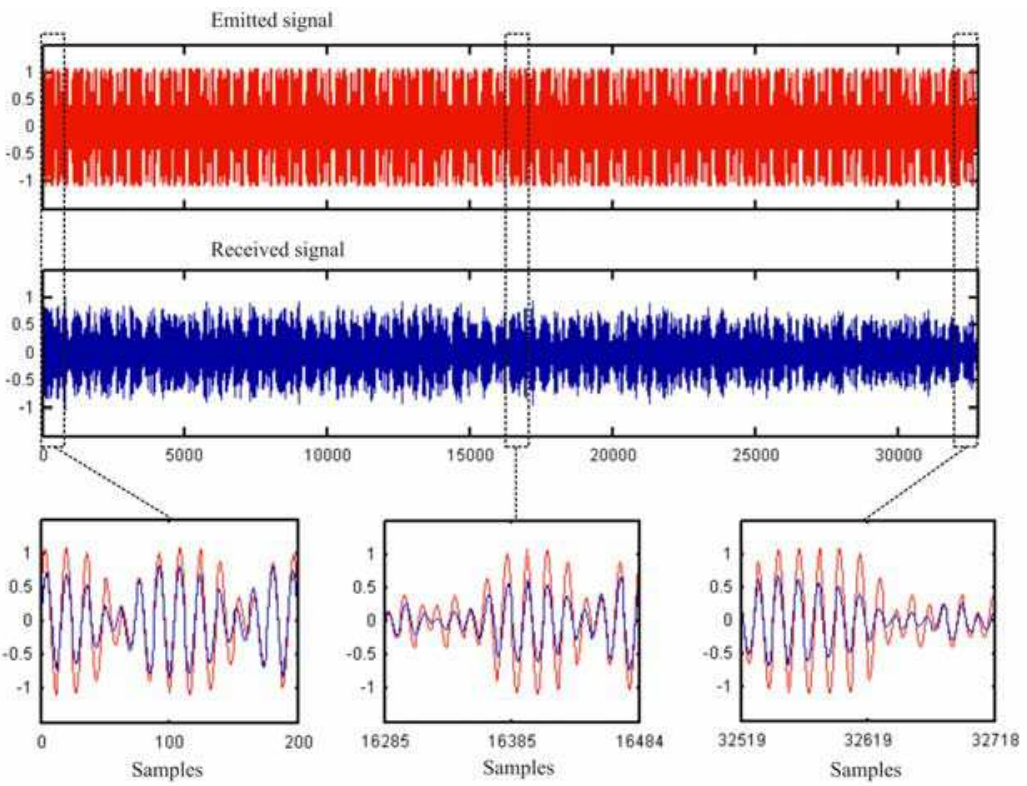

Fig. 14. Comparison between the emitted and the received signals under very weak turbulence conditions $(L=256)$.

under very strong turbulence, when the coherence time is clearly shorter than the emission time. In this case the average $S M R$ raises to 0.45 and, even more remarkable, the standard deviation is nearly three times this value. In this case, represented in Fig. 13b, very large sidepeaks are obtained whose height is occasionally higher than that of the corresponding main peak, given rise to values of $S M R$ above 1 . The system cannot reliably operate under these circumstances since these sidepeks could be erroneously validated as sets arrivals. 


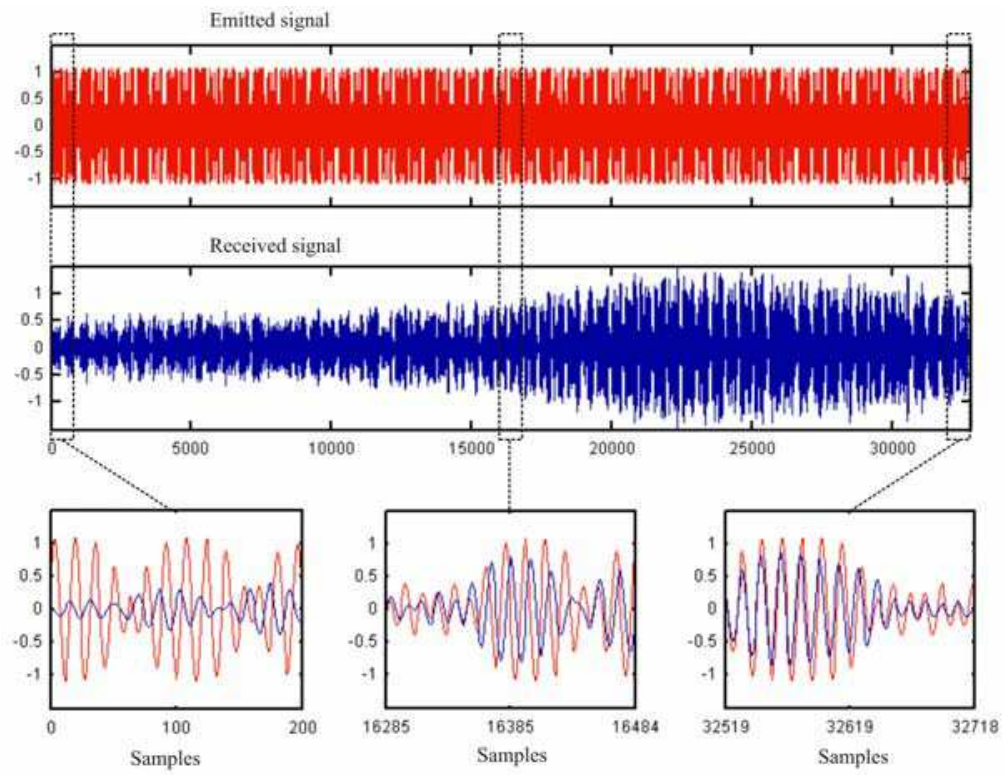

Fig. 15. Comparison between the emitted and the received signals under very strong turbulence conditions $(L=256)$.

\begin{tabular}{|c|c|c|c|c|}
\cline { 2 - 5 } \multicolumn{1}{c|}{} & \multicolumn{2}{c|}{64 bits } & \multicolumn{2}{c|}{256 bits } \\
\cline { 2 - 5 } \multicolumn{1}{c|}{ TURBULENCE } & Mean & Std & Mean & Std \\
\hline $\begin{array}{c}\text { Very weak } \\
\text { (cloudy, wind < 2m/s) }\end{array}$ & 0.1809 & 0.0225 & 0.1311 & 0.0268 \\
\hline $\begin{array}{c}\text { Weak } \\
\text { (sunny, wind <2m/s) }\end{array}$ & 0.1979 & 0.0441 & 0.1611 & 0.0660 \\
\hline $\begin{array}{c}\text { Medium } \\
\text { (cloudy, 2<wind <4 m/s) }\end{array}$ & 0.2032 & 0.0561 & 0.1630 & 0.0484 \\
\hline $\begin{array}{c}\text { Strong } \\
\text { (sunny, 2<wind <4 m/s) }\end{array}$ & 0.2396 & 0.1042 & 0.1924 & 0.0863 \\
\hline $\begin{array}{c}\text { Very Strong } \\
\text { Wind > 4 m/s }\end{array}$ & 0.2622 & 0.1289 & 0.4507 & 1.3012 \\
\hline
\end{tabular}

Table 1. Average Sidelobe-to-Mainlobe Ratio (SMR) under different turbulence conditions.

In addition to the appearance of spurious peaks, another phenomenon was observed during the experimentation. Although the arrivals of the sets are still correctly detected even when the coherence time is shorter than the emission time, the peaks associated to these arrivals appear more shifted from their expected positions with increasing turbulence intensity. The quantitative analysis of this phenomenon is summarized in Table 2. Note that when 64-bit sequences are transmitted, the average shift is always below $5 \mu$ s (4 samples!), although in all cases the dispersion is significant. The shift is even smaller with 256-bit sequences under similar conditions of turbulence, except again, when the coherence time is shorter than the emission time. In this case, the average shift jumps to nearly $60 \mu \mathrm{s}$ ( 3 carrier cycles) and the 
deviation goes up to $313 \mu \mathrm{s}$, showing that the actual values of shift vary wildly between a few $\mu$ s and several tenths of ms.

\begin{tabular}{|c|c|c|c|c|}
\cline { 2 - 5 } \multicolumn{1}{c|}{} & \multicolumn{2}{c|}{64 bits } & \multicolumn{2}{c|}{256 bits } \\
\cline { 2 - 5 } TURBULENCE & Mean $(\mu \mathrm{s})$ & Std $(\mu \mathrm{s})$ & Mean $(\mu \mathrm{s})$ & Std $(\mu \mathrm{s})$ \\
\hline $\begin{array}{c}\text { Very weak } \\
\text { (cloudy, wind < 2m/s) }\end{array}$ & 0.1762 & 0.4356 & 0.5842 & 0.6539 \\
\hline $\begin{array}{c}\text { Weak } \\
\text { (sunny, wind < 2m/s) }\end{array}$ & 0.2513 & 0.5016 & 0.4755 & 0.6377 \\
\hline $\begin{array}{c}\text { Medium } \\
\text { (cloudy, 2<wind <4 m/s) }\end{array}$ & 1.2304 & 4.0418 & 0.7609 & 0.7852 \\
\hline $\begin{array}{c}\text { Strong } \\
\text { (sunny, 2<wind <4 m/s) }\end{array}$ & 2.1508 & 5.5043 & 1.8503 & 2.2120 \\
\hline $\begin{array}{c}\text { Very Strong } \\
\text { Wind > 4 m/s }\end{array}$ & 4.8890 & 9.3993 & 55.927 & 313.42 \\
\hline
\end{tabular}

Table 2. Average shift of the autocorrelation peaks.

\section{Discussion and future research}

The design of high performance outdoor sonars seems to require the use of encoding and signal processing techniques to ensure the reliable operation of the system under changing meteorological conditions. This task introduces new problems mainly due to turbulence phenomenon and its random effect on the amplitude and phase of the emitted signals. We have seen that this effect can be characterized through a coherence time that is a measure of the time during which the features of the received signal remain essentially invariant. Some experimental results have been conducted showing that emissions below this time can be properly detected through matched filtering. Also, the deterioration of the correlated signal with decreasing coherence times has been verified by measuring the increase of its SMR and the autocorrelation peak shift.

Although we know that turbulence causes random variations on the amplitude and phase of acoustic signals, little is known about the statistical properties of these variations. An accurate model for this phenomenon would allow a precise prediction of the effects that a turbulent atmosphere has on the performance of advanced sonar systems where encoded signals are transmitted. It would also allow to clearly define the limits of operation for these systems. Moreover, this model could be used to determine the type of encoding and modulation schemes that would be more appropriate to operate under adverse conditions. Future research in this field should be focused on obtaining this model, as well as experimentally determining the accuracy of Eq. (31) that gives the coherence time dependence on frequency, turbulence intensity, normal component of wind and travelled distance.

\section{References}

Álvarez, F. J., Ureña, J., Mazo, M., Hernández, A., García, J. J. \& Jiménez, J. A. (2004). Efficient generator and pulse compressor for complementary sets of four sequences. IEE Electronics Letters, Vol. 40, No. 11 (May 2004), pp. 703-704. 
Álvarez, F. J., Ureña, J., Mazo, M., Hernández, A., García, J. J. \& De Marziani, C. (2006). High reliability outdoor sonar prototype. IEEE Transactions on Ultrasonics, Ferroelectrics and Frequency Control, Vol. 53, No. 10 (October 2006), pp. 1862-1871.

Álvarez, F. J., Ureña, J., Hernández, A., Mazo, M., García, J. J. \& Jiménez, A. (2008). Influence of atmospheric refraction on the performance of an outdoor pulse compression system. Applied Acoustics, Vol. 69, No. 11 (November 2008), pp. 994-1002.

Brown E. H. \& Clifford, S. F. (1976). On the attenuation of sound by turbulence. Journal of the Acoustical Society of America, Vol. 60, No. 4 (October 1976), pp. 788-794.

Clifford, S. F \& Brown, E. H. (1970). Propagation of sound in a turbulent atmosphere. Journal of the Acoustical Society of America, Vol. 48, No. 5 (November 1970), pp.1123-1127.

Cole, J. E \& Dobbins, R. A. (1970). Propagation of sound through atmospheric fog. Journal of the Atmospheric Science, Vol. 27, No. 3 (May 1970), pp. 426-434.

Golder, D. (1972). Relations among stability parameters in the surface layer. Boundary-Layer Meteorology, Vol. 3, No. 1 (September 1972), pp. 47-58.

Guo L., Zhang Q. \& Han S. (2002). Agricultural Machinery Safety Alert System Using Ultrasonic Sensors. Journal of Agricultural Safety and Health, Vol. 8, No. 4, (November 2002), pp. 385-396.

ISO/TC 43 Technical Comittee, Acoustics, Sub-Comittee SC1, Noise (1993). Attenuation of sound during propagation outdoors. Part 1: Calculation of the absorption of sound by the atmosphere, Tech. Rep. ISO 9613-1:1993 (E), International Organization for standarization (1993).

Jiménez A. R. \& Seco, F. (2005). Precise localisation of archaeological findings with a new ultrasonic 3D positioning sensor. Sensors and Actuators A, Physical. Vol. 123-124 (September 2005), pp. 224-233.

Kolmogorov, A. N. (1941). The local structure of turbulence in incompressible viscous fluid for very large Reynolds numbers. Proceedings of the USSR Academy of Sciences, Vol. 30, pp. 299-303.

Langer, D. \& Thorpe, C. (1992). Sonar based outdoor vehicle navigation and collision avoidance. Proceedings of IEEE/RSJ International Conference on Intelligent Robots and Systems, Raleigh, NC, (July 1992), pp. 1445-1450.

Maeyama S., Ohya A. \& Yuta S. (1994). Positioning by tree detection sensor and dead reckoning for outdoor navigation of a mobile robot. Proceedings of MFI'94, IEEE, Las Vegas, NV, (October 1994), pp. 653-660.

Monin A. S. \& Obukhov, A. M. (1954). Basic laws of turbulent mixing in the ground layer of the atmosphere. Transactions Geophys. Inst. Akad., Nauk USSR, Vol. 24, No. 151 (1954), pp. (163-187).

Ostashev, V. E. (1997). Acoustics in Moving Inhomogeneous Media. E \& FN SPON (An Imprint of Thompson Professional), London.

Panofsky, H. A. \& Dutton, J. A. (1984). Atmospheric Turbulence. Models and Methods for Engineering Applications. John Wiley \& Sons, Inc, New York, 1984

Polaroid Corporation (1999). 600 Series. Instrument Grade Electrostatic Transducers. Technical Specification, 1999.

Ratner D. \& McKerrow P. (2003). Navigating an outdoor robot along continuous landmarks with ultrasonic sensing. Robotics and Autonomous Systems, Vol. 45, No. 2 (November 2003), pp. 73-82. 
Shamanaeva, L. G. (1988). Acoustic sounding of rain intensity. Journal of the Acoustical Society of America, Vol. 84, No. 2 (August 1988), pp.713-718.

Tanzawa, T., Kiyohiro N., Kotani S. \& Mori K. (1995) The Ultrasonic Range Finder for Outdoor Mobile Robots. Intelligent Robots and Systems 95. 'Human Robot Interaction and Cooperative Robots', Proceedings. 1995. pp. 368-373. Pittsburgh, PA, USA.

Tatarskii, V. I. (1971). The Effects of the Turbulent Atmosphere on Wave Propagation. Israel Program for Scientific Translations, Ltd, Jerusalem, 1971.

Yura, H. T. (1971). Atmospheric turbulence induced laser beam spread. Applied Optics, Vol. 10, No. 12 (December 1971), pp. 2771-2773. 


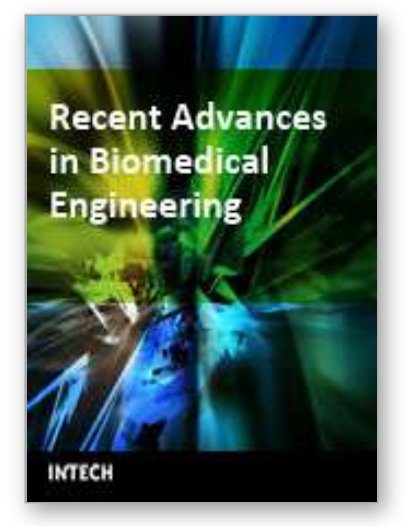

\author{
Advances in Sonar Technology \\ Edited by Sergio Rui Silva
}

ISBN 978-3-902613-48-6

Hard cover, 450 pages

Publisher I-Tech Education and Publishing

Published online 01, February, 2009

Published in print edition February, 2009

The demand to explore the largest and also one of the richest parts of our planet, the advances in signal processing promoted by an exponential growth in computation power and a thorough study of sound propagation in the underwater realm, have lead to remarkable advances in sonar technology in the last years. The work on hand is a sum of knowledge of several authors who contributed in various aspects of sonar technology. This book intends to give a broad overview of the advances in sonar technology of the last years that resulted from the research effort of the authors in both sonar systems and their applications. It is intended for scientist and engineers from a variety of backgrounds and even those that never had contact with sonar technology before will find an easy introduction with the topics and principles exposed here.

\title{
How to reference
}

In order to correctly reference this scholarly work, feel free to copy and paste the following:

Fernando J. Álvarez Franco and Jesús Ureña Ureña (2009). Outdoor Sonar Sensing, Advances in Sonar Technology, Sergio Rui Silva (Ed.), ISBN: 978-3-902613-48-6, InTech, Available from: http://www.intechopen.com/books/advances_in_sonar_technology/outdoor_sonar_sensing

\section{INTECH}

open science | open minds

\section{InTech Europe}

University Campus STeP Ri Slavka Krautzeka 83/A 51000 Rijeka, Croatia Phone: +385 (51) 770447

Fax: +385 (51) 686166 www.intechopen.com

\section{InTech China}

Unit 405, Office Block, Hotel Equatorial Shanghai No.65, Yan An Road (West), Shanghai, 200040, China 中国上海市延安西路65号上海国际贵都大饭店办公楼405单元 Phone: +86-21-62489820

Fax: +86-21-62489821 
(C) 2009 The Author(s). Licensee IntechOpen. This chapter is distributed under the terms of the Creative Commons Attribution-NonCommercialShareAlike-3.0 License, which permits use, distribution and reproduction for non-commercial purposes, provided the original is properly cited and derivative works building on this content are distributed under the same license. 